\title{
A critical analysis of the methodology for calculation of the Lighting Energy Numerical Indicator (LENI)
}

\author{
D. Gasparovsky ${ }^{1}$, E. Erkin ${ }^{2}$, S. Onaygil ${ }^{2} \&$ A. Smola ${ }^{1}$ \\ ${ }^{\text {I} S l o v a k ~ U n i v e r s i t y ~ o f ~ T e c h n o l o g y, ~ F a c u l t y ~ o f ~ E l e c t r i c a l ~ E n g i n e e r i n g ~ a n d ~}$ \\ Information Technology, Slovak Republic \\ ${ }^{2}$ Istanbul Technical University, Energy Institute, Turkey
}

\begin{abstract}
Energy certification and labelling of buildings according to the Commission's Energy Performance of Buildings Directive concerns four main energy consuming systems with lighting as one of them. Previously there was neither practice nor experience with certification of lightings systems. First practical experience brought a series of questions and problems to be solved on methodological level. Main goal of the paper is to treatise on identified problems with evidence shown on practical case studies. Imperfections in the light of stressed necessity to run the certification process evolved a big amount of research works performed in order to improve the methodology and to investigate energy saving potential in buildings with new approaches. For each individual problem a solution is developed, which is now already implemented to the legislation on national level and put in practice. It is expected that these solutions may help to improve the current methodology by revision of the standard and this way to become a broader acceptance. Practical experience will help to support this effort. Solutions are introduced in this paper.

In final part of the paper, a software tool developed for calculation of LENI is presented. Important feature of the software named EHB LiteCalc is that all steps of calculation can be separately inspected, also giving the user possibility to enter the calculation process by inputting manually forced values. This special option can serve for experimental purposes.
\end{abstract}

Keywords: energy efficiency, energy performance of buildings, lighting energy, LENI. 


\section{Introduction}

With the rapid increase of human population resulting in higher energy demands day by day, critical level of greenhouse gas emissions (particularly $\mathrm{CO}_{2}$ ) has already been reached. Thus, many studies, actions and implementation plans have intensively been realized for more than ten years. Since it is a global threat, the Kyoto Protocol which is a protocol of the United Nations Framework Convention on Climate Change (UNFCCC), was adopted in 1997 and entered into force in 2005 with the goal of achieving stabilization of greenhouse gas concentrations in the atmosphere at a level that would prevent dangerous anthropogenic interference with the climate system $[1,2]$.

In the EU political and economical area, several directives referring to the Kyoto protocol aiming to the reduction of greenhouse gases have already been published. European Parliament and the Council adopted the Directive on Energy Performance of Buildings (EPBD) 2002/91/EC [3] on December 16, 2002. Main goal of the directive is to improve the energy efficiency of buildings. Four main areas have been identified, where lighting systems play an important role - as in lighting there is huge energy saving potential expected due to very fast technological development.

EU member countries were obliged to implement this important directive into national legislation not later than January 4, 2006. National Parliament of the Slovak Republic fulfilled its obligation by release of the Act No. 555/2005 [4], prepared by the Ministry of Construction and Regional Development under tight collaboration with experts responsible for individual energy systems concerned. Requirements for lighting and issues relevant to lighting have been prepared with participation of authors of this paper. Basic methodological principles, having force of legislation, are today given by the Ordinance No. 311/2009 [5]. By this ordinance, practical experience and identified problems and difficulties with certification, described in this paper, have been reflected by relevant solutions. Technical details of the methodology for lighting are covered by EN 15 193:2007 [6]. Although there are many considerations on lighting systems and building properties related to lighting in this standard, some problems occurred during implementation process are necessary to be solved in order to make its best implementation in practice and to achieve accurate and comparable results. Slovak Republic developed his own national methodology [7] introducing new approaches to some of the relevant problems. Turkey also prepared his national methodology, which came in force by December 5th, 2009. Bringing the EPBD and EN 15193 in particular into practice is the subject of a joint effort within the European CENSE project [8].

\section{Theoretical background}

The European standard EN 15193 was devised to establish conventions and procedures for the estimation of energy requirements of lighting in buildings, and to give a methodology for a numeric indicator of energy performance of buildings used for certification purposes. It also provides guidance on the 
establishment of notional limits for lighting energy derived from reference schemes.

The standard can be used for existing buildings and for the design of new or renovated buildings. It also provides reference schemes to base the targets for energy allocated for lighting usage and also provides a methodology for the calculation of instantaneous lighting energy use for the estimation of the total energy performance of the building.

Energy consumption related to building area is defined as a Lighting Energy Numeric Indicator (LENI) in $\mathrm{kWh} /\left(\mathrm{m}^{2}\right.$.year), which can be established using the following equation:

$$
L E N I=\frac{W_{L}+W_{P}}{A}
$$

where

$\mathrm{W}_{\mathrm{L}} \quad$ - lighting energy for illumination $(\mathrm{kWh} /$ year)

$\mathrm{W}_{\mathrm{P}}$ - parasitic energy $(\mathrm{kWh} /$ year $)$

A - total useful floor area of the building $\left(\mathrm{m}^{2}\right)$

Lighting energy required for fulfilling the illumination function and purpose in building shall be established using the following equation:

$$
W_{L}=P_{n} F_{C} F_{O}\left(t_{D} F_{D}+t_{N}\right)
$$

where

$\mathrm{P}_{\mathrm{n}} \quad$ - installed power of the lighting system $(\mathrm{kW})$

$\mathrm{F}_{\mathrm{D}} \quad$ - daylight dependency factor (-)

$\mathrm{F}_{\mathrm{O}} \quad$ - occupancy dependency factor (-)

$\mathrm{F}_{\mathrm{C}} \quad$ - constant illuminance factor (-)

$t_{D} \quad$ - daylight time usage $(\mathrm{h})$

$\mathrm{t}_{\mathrm{N}} \quad$ - non-daylight time usage $(\mathrm{h})$

Parasitic energy is estimated using equation that incorporates total installed parasitic power for standby energy of the controls and charging power of the emergency luminaries regarding the charging time of emergency luminaries. Details can be found in the norm [6].

\section{Identified problems and recommended solutions}

\subsection{Insufficient lighting levels}

Having the correct lighting standard in buildings has utmost importance and the convention and procedures assume that the designed and installed lighting scheme conforms to good lighting practice. For new installations withindoor work places the design should be with respect to EN 12464-1 [9]. Thus, in order to compare buildings between each other, estimated energy consumption of lighting systems should be determined in all buildings that are assumed to fulfill the lighting criteria particularly the average illumination level. If this cannot be satisfied, then required energy demand for lighting will be considerably lower. 
This situation may mislead the certification process into inconvenient situation that will prevent the comparison possibilities between buildings. Therefore within certification it is necessary to check illumination levels according to relevant technical standards.

As a common approach, the energy consumption values can be normalized to values that can provide required illumination levels. One of the proposed approaches how to avoid invalidity of assessments is based on an additional Maintained Illuminance Factor $\mathrm{F}_{\mathrm{em}}$ which is introduced to the Eq. (2) aside other factors. Now the Eq. (3) becomes a new form as follows:

$$
F_{E m}=\frac{E_{r}}{E_{m}}
$$

where:

$\mathrm{E}_{\mathrm{r}} \quad$ - required illuminance level (lx)

$\mathrm{E}_{\mathrm{m}} \quad$ - measured illuminance level (lx)

Note, that the maintained illuminance factor is more than one for buildings (or rooms) with insufficient lighting. If measured illuminance is higher than required, the maintained illuminance factor is set to $\mathrm{F}_{\mathrm{Em}}=1=$ const. $\mathrm{F}_{\mathrm{Em}}$ is to be determined by proper measurements of selected rooms with emphasize on illumination of workplaces. The following rules shall apply:

- If possible, calculations should cover all rooms of a building. Then $\mathrm{F}_{\mathrm{Em}}$ values and $\mathrm{W}_{\mathrm{L}}$ shall be calculated for each room separately.

- Otherwise rooms shall be sorted descending according to installed power.

- Rooms shall be selected for verification until representing at least $50 \%$ of the total installed power.

- Rooms shall be selected for verification considering the usage time; rarely used rooms should be ignored.

- If there is enormous number of rooms in a building, similar rooms shall be taken as duplications. In addition, $20 \%$ of rooms can be considered as a sufficient sample for validation of lighting criteria.

- Average $\mathrm{F}_{\mathrm{Em}}$ values shall be calculated for the selected and measured rooms and used to multiply $\mathrm{W}_{\mathrm{L}}$ for the building as a whole.

Certainly, there can be several approaches to reduce the number of measurements bringing a selective modality to assign the rooms to be measured. Since installed power is mentioned as a selective parameter together with usage time, third bullet of the list above applies. If there are several rooms with lower areas, it can still be unpractical to achieve the $50 \%$ level and therefore the $20 \%$ sample under the fifth bullet shall be preferred. Note that proposed ratios are draft values and should be determined by experimental and statistical approach on national level.

A different kind of approach is to introduce a punitive factor similar to $\mathrm{F}_{\mathrm{Em}}$, with a constant value. This approach is implemented in the Slovak National Methodology [7] where a factor of three is used. This number comes from practical experience with hygienic measurements of illumination of workplaces (a legislative requirement for successful commissioning of buildings) and 
auditing of older buildings. Statistical evaluation of numerous measurement results showed that, in average, illuminance is only about one third of the current normative requirement. Sounds to be suspiciously low, but this is an everyday reality for both of old existing buildings as well as new constructions. As results of small survey performed in last year showed, lighting designers almost do not exist here and their job is taken by designers of electrical installations who are not skilled for the task, then they mainly draw from what they can see in older buildings, also what the illumination levels concerns. The least efficient solutions with rectangular modular $4 \times 18 \mathrm{~W}$ fluorescent lamps are most popular even for the most demanding and representative interiors. Hence the factor of three.

This approach has been implemented in time when the mentioned problem was just identified and quick solution was needed. Approach with $\mathrm{F}_{\mathrm{Em}}$ corrections took place in later stage. However, a punitive approach needs not to be deemed as too rough. To fulfill light quality criteria is a normative obligation with corresponding responsibility. If measurements in arbitrary selected rooms give evidence of violation of these requirements, it is not the task of certificant to evaluate as accurate as possible correction factor in order to come closer to generally acceptable results. Thus, lighting system may obtain good ranks if incorporating energy efficient solutions although do not duly fulfill its lighting task. Punitive approach will always point to bad systems and force investor or building proprietor to make improvements, followed by new certification process. This is in scope of the Directive.

Let us have now a closer look to the punitive approach and its procedural aspects, as these have been fully elaborated by authors of this paper. Verification method should be undoubtedly based on measurements; visual inspection can lead to excessive subjective errors. For the purpose, simplified measurement methods may take place. If e.g. for the commissioning of buildings protocols of (much more precise) measurements are available, these can be fully regarded. Otherwise certificant should select $10 \%$ of the total number of rooms for measurement as a minimum. Details are not prescribed; it is assumed that certificant is a high-skilled expert who is able to make a good choice. It is necessary to mention that the method has been proposed as a legislative tool for certificant helping him to avoid ranking high those lighting systems that are under dimensioned. Illuminance levels should be checked in sufficient number of measurement points and on critical places, points need not to be arranged to a grid. $10 \%$ of all measured rooms may still fail to fulfill the criteria.

Having in mind pros and cons of $\mathrm{F}_{\mathrm{Em}}$ approach vs. punitive approach, it is expected that the first will substitute the latter in Slovak national method by its next revision. Further research works are still needed here.

\subsection{Quick method usability}

The quick method is intended for lighting design. Its simplification versus the comprehensive method naturally leads to higher values of energy consumption, what is also mentioned in the European standard EN 15193. As a consequence of the quick method philosophy, calculations need not to be performed room by 
room but for building as a whole what is a significant simplification. Thus, total installed power of lighting systems in a building is taken as a single number.

Lighting installation usage times $t_{D}$ and $t_{N}$ for daylight and non-daylight operation hours have critical influence to the resulting value (of consumption and thus for LENI as well) but the norm do not provide any standardized method how to calculate them (further explanation can be found in Section 3.3). Instead, for the quick method standard tabelized values are provided. Their relevance is discussed in Section 3.4.

Factors $\mathrm{F}_{\mathrm{D}}, \mathrm{F}_{\mathrm{O}}$ and $\mathrm{F}_{\mathrm{C}}$ help to reduce the energy consumption for lighting assuming that if there is available daylight or if rooms are not occupied, eventually if a system compensating the MF is installed; lighting is not working on $100 \%$ in the whole building and during the whole operation time. It can be expected that influence of these factors is very critical. Using the comprehensive method, these factors are to be obtained by calculation. For the quick method, benchmark values are provided, though, both $\mathrm{F}_{\mathrm{D}}$ and $\mathrm{F}_{\mathrm{O}}$ are not too far from 1 and manually operated systems have these factors always equal to 1 . As a results, energy consumption $\mathrm{W}_{\mathrm{L}}$ and/or LENI are then much higher than by using the comprehensive method. As in Slovak Republic the energy efficiency classes are defined separately also for lighting (and all other sub-systems as well), usage of the quick method leads to a difference of three or even more classes in comparison to the comprehensive method while we can desire the maximum one class difference.

It can be concluded that using the normative benchmark values makes the quick method unusable. Therefore, in Slovakia national standard values of $\mathrm{F}_{\mathrm{D}}, \mathrm{F}_{\mathrm{O}}$ and $F_{C}$ factors have been established (see Tables 1 and 2), derived from statistical evaluation of buildings assessed by the comprehensive method, i.e. averaging the factors from room-per-room values. It is evident that considering full operation of lighting with manual control is an overestimation. In any building, besides main rooms with workplaces, there is a significant portion of auxiliary rooms like toilets, washrooms, corridors, stairs and a lot of storage rooms where almost nobody enters during all the day. It is not smart to take fulltime operation of such rooms even if manually controlled. The same is for daylight availability. Even if no light sensor is installed and lighting is controlled manually, at sufficient daylight occupants do not switch-on their lighting. Automated systems undoubtedly help to reduce power but manual control must not be underrated.

Parasitic power is another question for consideration. The quick method offers, where the parasitic energy consumed is not known explicitly, standard values consisting of $1 \mathrm{kWh} /\left(\mathrm{m}^{2}\right.$.year $)$ for emergency lighting plus $5 \mathrm{kWh} /\left(\mathrm{m}^{2}\right.$.year) for automatic lighting controls if used. As it was shown in practice, the mentioned figures are much overestimated and undesirably contribute to high consumptions. First, if there is no any emergency lighting or lighting control in a building, $\mathrm{W}_{\mathrm{P}}$ should be taken as zero. Else it needs to be either calculated like the installed power or to use nationally derived standard values. In Slovakia, standard value based on practical experience has been set to 0,5 . 
Table 1: $\quad$ Lighting control strategies.

\begin{tabular}{|l|c|}
\hline Lighting control & Group \\
\hline Manual: ON/OFF without sensors & R1 \\
\hline Manual: ON/OFF with sweeping signal & R2 \\
\hline Motion detector: auto ON + dimming & R3 \\
\hline Motion detector: auto ON + auto OFF & R4 \\
\hline Motion detector: manual ON + dimming & R5 \\
\hline Motion detector: manual ON + auto OFF & R6 \\
\hline Photo sensor: manual ON + constant illuminance dimming & R7 \\
\hline Photo sensor: daylight control switching or dimming & R8 \\
\hline Central control & R9 \\
\hline
\end{tabular}

Table 2: $\quad$ Slovak national values of daylight factor $F_{D}$ and occupancy factor $\mathrm{F}_{\mathrm{O}}$ for the quick method.

\begin{tabular}{|l|c|c|c|c|c|c|c|}
\hline Factor & \multicolumn{3}{|c|}{$\mathrm{F}_{\mathrm{D}}$} & \multicolumn{4}{c|}{$\mathrm{F}_{\mathrm{O}}$} \\
\hline Building category & $\begin{array}{c}\mathrm{R} 1- \\
\mathrm{R} 7\end{array}$ & $\mathrm{R} 8$ & $\mathrm{R} 9$ & $\begin{array}{c}\text { R1 }- \\
\mathrm{R} 2\end{array}$ & $\begin{array}{c}\text { R3 }- \\
\text { R6 }\end{array}$ & $\begin{array}{c}\mathrm{R} 7- \\
\mathrm{R} 8\end{array}$ & $\mathrm{R} 9$ \\
\hline Office buildings & 0,92 & 0,85 & 0,92 & 0,7 & 0,5 & 0,7 & 0,6 \\
\hline Schools and edu buildings & 0,92 & 0,85 & 0,92 & 0,5 & 0,4 & 0,5 & 0,5 \\
\hline Hospitals & 0,92 & 0,90 & 0,92 & 0,9 & 0,9 & 0,9 & 0,9 \\
\hline Hotels & 0,92 & 0,92 & 0,92 & 0,8 & 0,7 & 0,8 & 0,8 \\
\hline Restaurants & 0,98 & 0,98 & 0,98 & 1,0 & 1,0 & 1,0 & 1,0 \\
\hline Sport facilities & 1,00 & 1,00 & 1,00 & 1,0 & 1,0 & 1,0 & 1,0 \\
\hline Wholesales and shops & 1,00 & 1,00 & 1,00 & 1,0 & 1,0 & 1,0 & 1,0 \\
\hline
\end{tabular}

\subsection{Calculation of daylight time and non-daylight time usage}

Comprehensive method requires determination of annual daylight and nondaylight time usage for calculation of the estimated energy demand of a building. There had been defined a methodology integrated in the first draft of the standard EN 15193, but composed equations were nonfunctional, hence excluded from the final version of this standard. As a result, there is no guideline for authorized certificants how to determine the daylight time and non-daylight time usage.

The proposed method is inspired by original procedure of the draft version of EN 15193 and Slovak studies utilizing experience in the field of solar energy engineering, involving various approximation formulae. This method is standardized within the Slovak National Methodology and with slight modifications adopted also for Turkish conditions. The calculations are proposed on a monthly basis.

Annual daylight time usage $t_{D}\left(h /\right.$ year) and non-daylight time usage $t_{N}(h /$ year $)$ is calculated by summing up the respective monthly values $t_{\mathrm{D}, \mathrm{I}}$ and $\mathrm{t}_{\mathrm{N}, \mathrm{i}}$ :

$$
t_{D}=\sum_{i=1}^{12} t_{D, i} t_{N}=\sum_{i=1}^{12} t_{N, i}
$$


Daylight time usage $t_{D, i}(h / m o n t h)$ and non-daylight time usage $t_{N, i}(h / m o n t h)$ for a given month " $i$ " can be calculated as follows:

$$
\begin{gathered}
t_{D, i}=N_{i} C_{w e}\left[\left(t_{\text {end }}-t_{\text {start }}\right)-\left(t_{b s, i}+t_{a s, i}\right)\right] \\
t_{N, i}=N_{i} C_{w e}\left[\left(t_{b s, i}+t_{a s, i}\right)\right]
\end{gathered}
$$

where:

$\mathrm{N}_{\mathrm{i}} \quad$ - number of days for a given month (monthly basis)

$\mathrm{C}_{\mathrm{we}} \quad$ - correction factor for weekends (-)

$\mathrm{t}_{\text {start }} \quad$ - starting operation time of a building (FROM)

$\mathrm{t}_{\mathrm{end}} \quad$ - ending operation time of a building (TO)

$t_{b s, i} \quad$ - operation time before sunrise (h/day)

$\mathrm{t}_{\mathrm{as}, \mathrm{i}} \quad$ - operation time after sunset (h/day)

Note that starting and ending operation times are now standardized in Slovakia, tailored for each building category (see Table 3). Number of days for a given month " $i$ " (1 to 12$)$ is taken from this set:

$\mathrm{N}_{\mathrm{i}}=\{31,28,31,30,31,30,31,31,30,31,30,31\}$

Weekend regime is regarded by means of the weekend correction factor $\mathrm{C}_{\mathrm{we}}$ which is a ratio of working days to full week's 7 days. Time of sunrise and sunset are calculated by means of these equations:

$$
\begin{aligned}
& t_{\text {sunrise }, i}=12-\frac{\omega_{i}}{15^{\circ}}-\eta\left(\frac{J_{i}}{60}\right) \\
& t_{\text {sunset }, i}=12+\frac{\omega_{i}}{15^{\circ}}-\eta\left(\frac{J_{i}}{60}\right)
\end{aligned}
$$

where:

$\mathrm{J}_{\mathrm{i}} \quad-$ order number for $15^{\text {th }}$ day of a given month " $\mathrm{i}$ "

$\omega_{\mathrm{i}} \quad$ - hour angle $\left(^{\circ}\right)$

$\eta_{\mathrm{i}} \quad$ - time equation $\left(^{\circ}\right)$

Order number for $15^{\text {th }}$ day of a given month " $i$ " ( 1 to 12$)$ is taken from this set: $\mathrm{J}_{\mathrm{i}}=\{15,46,74,105,135,166,196,227,258,288,319,349\}$.Time equation $\eta_{\mathrm{i}}$ can be calculated by means of the formula as follows:

$$
\begin{aligned}
& \eta(J)=0,0066+7,3525 \cos \left(J^{\prime}+85,9^{\circ}\right)+9,9359 \cos \left(2 J^{\prime}+108,9\right) \\
& +0,3387 \cos \left(3 J^{\prime}+105,2\right)
\end{aligned}
$$

where:

$\mathrm{J}^{\prime}=\mathrm{J} .360^{\circ} / 365$ isdate angle $\left(^{\circ}\right)$

For calculation of hour angle $\omega_{\mathrm{i}}$, the following equation is available:

$$
\omega_{i}=\arccos \left[-\frac{\sin \varphi \sin \left(J_{i}^{\prime}\right)}{\cos \varphi \operatorname{sos}\left(\delta J_{i}\right)}\right]
$$


where:

$\delta_{\mathrm{i}} \quad-\operatorname{solar}$ declination $\left(^{\circ}\right)$

$\varphi \quad$ - geographical latitude of a building $\left(^{\circ}\right)$

Declination $\delta_{\mathrm{i}}$ can be calculated as follows:

$$
\begin{aligned}
& \delta(J)-0,3948-23,2559 \cos \left(J^{\prime}+9,1^{\circ}\right)-0,3915 \cos \left(2 J^{\prime}+5,4^{\circ}\right) \\
& +0,1764 \cos \left(3 J^{\prime}+26,0^{\circ}\right)
\end{aligned}
$$

Procedures given above are algorithmized and standardized in the Slovak National Methodology. The idea was to keep the philosophy of the first proposal of EN 15193, which finally has not been published, and to make corrections in order to satisfy the functionality and to obtain valid results. To ensure standardization, starting and ending time of building operation have been set constant in dependence on building category, but unfortunately $\mathrm{C}_{\mathrm{we}}$ was omitted from this standardization. Certificants now experience confusedness in practice, using different weekend correction factor with a significant influence to results.

For Turkish conditions, Slovak National Methodology has been used as a basement. Modifications to some of the formulae like (7), (8) and (11) have been made. Improvements incorporate also the daylight saving time. As a next step, further development of the Slovak National Methodology is under preparation. According to new proposals, calculation of declination and time equation are based on approximation formulae by Dogniaux in Kittler and Mikler [10]. Daily calculation basis is preferred now against the monthly basis. Average monthly values can be still used for the sake of simplicity but now calculated by new formulae. Previous philosophy of the draft EN 15193 is released. It is expected that new procedures will increase the accuracy of calculations and will be tailored to the European conditions.

What concerns the $\mathrm{C}_{\mathrm{we}}$ factor, new approach is studied. Before, this correction factor only adjusted the weekly operating time by ratio of the number of working days over 7 days of the week. This approach seems to be too rough. Operation during Saturday and Sunday much differs from operation behavior during normal working weekdays. While most of the buildings (administrative, educational) have reduced weekend operation (if any) while other building types may have emphasize right on weekends - like some sport facilities or shopping malls. Thus, a different approach is needed here.

\subsection{Determination of building operating hours}

As mentioned before in Section 3.2, operation hours are closely linked to the energy consumption. The standard EN 15193 encourages us to use real operating hours of buildings in calculations. Turning attention to objectives of the Directive, such approach cannot be accepted. Aim of the efforts is to compare buildings of similar functionality between each other what concerns their energy demand. Only and only technical properties of buildings shall to be considered, in no case the behavior of their occupants. 
An example can explain how serious this problem is. Let us have two buildings of the same functional usage, of the same construction and dimensions, with the same type of lighting system etc. The only difference will be in operating time. While first of the buildings will be operated e.g. since 7 am to 16 $\mathrm{pm}$, the other one will have non-stop all-day-round operation. This situation was noticed amongst first certificates made in Slovakia, 24h-operation was in a dispatcher centre of a natural gas provider. Of course, as expected, the energy consumption for lighting in non-stop regime was three times the consumption in the other building, therefore with very bad ranking ( $\mathrm{G}$ class). But properties of buildings are to be labelled, not if the building is used part of day or whole day. For this reason, operation times have to be standardized regardless on real usage times. May be such a way energy consumption will not be a precise realistic value, what is not the aim indeed, rather it will be a value comparable to other similar buildings calculated the same way and under the same standard conditions.

To give a solution, operation times have been standardized. For the quick method, annual operation times have been provided. For the comprehensive method, standard operation times are given in a FROM - TO format, necessary for daily and monthly based calculations (Table 3).

Table 3: Default annual operating hours for the quick and comprehensive method.

\begin{tabular}{|l|c|c|c|c|c|}
\hline Method & \multicolumn{3}{|c|}{ Quick } & \multicolumn{2}{c|}{ Comprehensive } \\
\hline Building category & $\mathrm{t}_{\mathrm{D}}$ & $\mathrm{t}_{\mathrm{N}}$ & $\mathrm{t}_{\mathrm{O}}$ & FROM & $\mathrm{TO}$ \\
\hline Office buildings & 2250 & 250 & 2500 & $7: 00$ & $16: 30$ \\
\hline $\begin{array}{l}\text { Schools and educational } \\
\text { buildings }\end{array}$ & 1800 & 200 & 2000 & $7: 00$ & $14: 30$ \\
\hline Hospitals & 3000 & 2000 & 5000 & $7: 00$ & 21.00 \\
\hline Hotels & 3000 & 2000 & 5000 & $7: 00$ & $22: 00$ \\
\hline Restaurants & 1250 & 1250 & 2500 & $7: 00$ & $22: 00$ \\
\hline Sport facilities & 2000 & 2000 & 4000 & $7: 00$ & $20: 00$ \\
\hline Wholesale and shops & 3000 & 2000 & 5000 & $7: 00$ & $20: 00$ \\
\hline
\end{tabular}

\section{Development of software tools to aid calculations}

Complicatedness of methodology does not allow calculation other than by means of computeral tools as all the procedures must be performed for each individual room. Due to differences between national conditions, mainly country specific software is being created. Philosophy is based on the idea of normative methodological core and adjustment of national databases and conditions.

Another question is if there should be common software for all sub-systems or three-four independent programs for experts active in their field. It seems, and first experience give a clear evidence, that independently acting experts have just a little chance to meet using one tool, what is unpractical and time consuming. Independent software are therefore prepared instead. In Slovak Republic the state 
of the art follows this philosophy and it is supposed to possibly tie these programs together in future via generally agreed data exchange format (some R\&D work is still needed here).

Authorisation of software is also a matter of discussions. The question is, if there should be a body responsible for verification of commercially manufactured software or there will be no guarantee for the software usage. Current decisions made in Slovakia follow the principle of liberal market without authorisation. It means, in fact, that software must be assumed as a tool for certificants, while certificants are fully responsible for their results. It practically means just one - certificants must be provided for all the interim results, they need to have an option to watch, check, inspect and modify every single result throughout the calculation procedure. And software has to allow this.

EHB LiteCalc (comprehensive method) and EHB QuickCalc (quick method) [11], developed by Typhoon, are examples of software supportive tools having these features. EHB LiteCalc (actually in version 3) is based upon well known and highly accepted excel format what makes it very easy to use. The newest version is available with improvements of calculation procedures and user environment. It should ease the work of certificants and to provide new options. Currently the software has implementation of Slovak and Turkish methodology, Czech version is under preparation and there is possibility to extend this software to any other national conditions. For general usage there is English version available with basic normative methodology (free of national modifications).

\section{Conclusions}

Energy certification of buildings is still an unmatured process. Most of work is still up to come as problems and imperfections arise from current practical experience with certification. This creates a big amount of research works to be performed, in order to improve the methodology and to investigate energy saving potential in buildings, to find out new approaches - all this is a challenge for all who want to add a value to the process leading towards such important aims like limitation of greenhouse effects and climate change.

Insufficient lighting is one of the most important problems that mislead the certification process and need to be considered in every adoption process. Quick method is found as unusable with normative benchmark values. As the standard excludes procedure for determination of daylight and non-daylight usage time of buildings, national methodology should be offered as a guideline which is a need for majority of certificants.

Slovak National Methodology is a pioneering document drawing from conscious approach to the practical implementation of the Directive. It is a very positive fact that the field of lighting was let to lighting experts, unlike in series of other countries where all fields are covered by civil engineers with lacking knowledge of lighting. What concerns the methodology itself, experts from other countries may learn from the experience though national adjustment of criteria may be needed. And vice versa, there are still a lot of questions to be solved and even current solutions are still open to further improvements. 
Turkey recently developed his national methodology as well. It incorporates many improvements of previously available methods that have been studied in the preparatory phase. First certificates and audits will confirm if national conditions have been correctly set. There is also big potential for further improvements and for significant contribution to the continuous development of the European methodology.

\section{Acknowledgement}

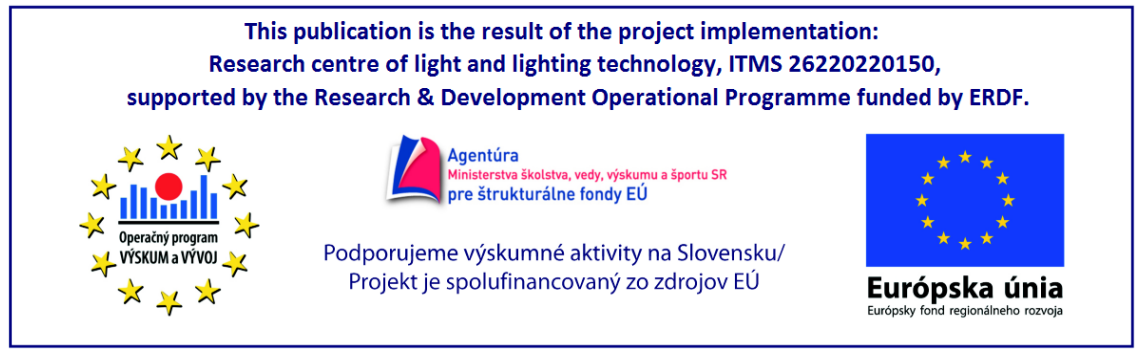

\section{References}

[1] United Nations Framework Convention on Climate Change. UN: Rio, 1992

[2] Kyoto Protocol. UN: Morocco, 1997

[3] Directive of the European Parliament and of the Council on Energy Performance of Buildings. Brussels, 2006

[4] Act No. 555/2005 of the National Parliament of Slovak Republic on the Energy Performance of Buildings. Bratislava, 2005

[5] Ordinance No. 311/2009 of the Ministry of Construction and Regional Development of Slovak Republic. Bratislava, 2009

[6] EN 15 193:Energy performance of buildings — Energy requirements for lighting, 2007

[7] Slovak National Methodology on Energy Performance of Buildings

[8] Staudt, A.-deBoer, J.- Erhorn, J.: CENSE: A joint effort on bringing the EPBD and CEN 15193 "prEN 15193: Energy performance of buildings Energy requirements for lighting" into practice. In Proc: Lux Europa 2009. Turkish National Committee on Illumination: Istanbul, pp. 571 - 578, 2009

[9] EN 12464-1:Light and lighting. Lighting of work places. Indoor work places, 2002

[10] Kittler, R. \& Mikler, J.: Fundamentals of the solar radiation utilization. VEDA: Bratislava, pp. 27 - 40, 1986 (in Slovak)

[11] Software package EHB LiteCalc 3.1, EHB QuickCalc 3.1, Typhoon: Bratislava, 2010 